\title{
Predictive value of some pro-oxidants in type 2 diabetes mellitus with vascular complications
}

\author{
Petia Goycheva $^{1, *}$, Galina Nikolova ${ }^{2}$, Mariana Ivanova $^{3}$, Todor Kundurdzhiev ${ }^{4}$, \\ Veselina Gadjeva ${ }^{2}$ \\ ${ }^{1}$ Clinic of Endocrinology, University Hospital "Prof. Dr. Stoyan Kirkovich", Medical Faculty, Trakia University, Stara Zagora, \\ Bulgaria; \\ ${ }^{2}$ Department of Chemistry and Biochemistry, Medical Faculty, Trakia University, Stara Zagora, Bulgaria; \\ ${ }^{3}$ Clinic of Rheumatology, University Hospital "St. Ivan Rilski", Medical Faculty, Medical University, Sofia, Bulgaria; \\ ${ }^{4}$ Faculty of Public Health, Medical University, Sofia, Bulgaria.
}

\begin{abstract}
Summary The study aims to analyze oxidative stress levels in circulation of some reactive molecules and products of biomolecular modification in type 2 diabetes mellitus (T2DM) with diabetes-specific vascular complications in order to determine their predictive value. Also, the alterations of their serum concentration with reference to disease characteristics were assessed. Reactive oxygen species (ROS), nitric oxide radicals $(\bullet \mathrm{NO})$, malondialdehyde (MDA), protein carbonyl (CO) and 8-hydroxydeoxyguanosin (8-OHdG) in serum were measured in 93 patients with T2DM with vascular complications, 94 control subjects and 16 diabetic patients who had no evidence of vascular disease. T2DM patients with clinically manifest vascular disease exhibit significantly elevated concentrations of all pro-oxidants in comparison to healthy subjects, with the highest degree of increase of $\bullet \mathrm{NO}$ radicals. The levels of carbonylated proteins, ROS and 8-OHdG were significantly increased in insufficiently compensated diabetes as compared to good glycemic control state. Also, serum MDA, protein $\mathrm{CO}$ and 8-OHdG showed an association with glycemic control parameters. MDA, ROS and 8-OHdG correlated mostly with microvascular complications. Significant area under the curve (AUC) from plotted receiver operating characteristic (ROC) curves were obtained for all studied biomarkers, as for nitric oxide it was substantially bigger compared to those for the other pro-oxidants. Correspondingly, positive and negative predictive values related to the disease were in favor of the $\bullet \mathrm{NO}$ radicals. The cutoff values of oxidative biomarkers may serve as an indicator of clinical reference for detecting T2DM with associated vascular complications, as nitric oxide radicals were the most reliable indicator.
\end{abstract}

Keywords: Type 2 diabetes mellitus, diabetes-specific vascular complications, oxidative stress, pro-oxidants

\section{Introduction}

Type 2 diabetes mellitus (T2DM) is a metabolic disease characterized by hyperglycemia and dyslipidemia due

Released online in J-STAGE as advance publication April 8, 2019.

*Address correspondence to:

Dr. Petia Goycheva, Clinic of Endocrinology, University Hospital "Prof. Dr. St. Kirkovich", 11 Armeyska Str., Stara Zagora 6003, Bulgaria.

E-mail: petya_goicheva@yahoo.com to pancreatic $\beta$-cell dysfunction and insulin resistance. Usually, insulin resistance of target tissues such as the liver, muscles and fat stores precedes the onset of T2DM by many years, resulting in an initial hyperinsulinemia and followed by a consequent deficiency of insulin secretion by pancreatic beta cells. Impaired insulin secretion by the islet cells and impaired insulin action through insulin resistance are the hallmarks in type 2 diabetes (1). The disease is largely responsible to macrovascular (coronary heart disease, peripheral vascular disease and stroke), microvascular (neuropathy, retinopathy and nephropathy) and both micro- and 
macrovascular (diabetic foot) complications, which are the main reason for the mortality and morbidity (2).

It has been shown that oxidative stress via the production of larger amounts of reactive oxygen species (ROS) is implicated in the progression of insulin resistance, pancreatic $\beta$-cell dysfunction as well as in vascular degeneration processes and the accelerated atherosclerosis in T2DM $(3,4)$. The metabolic abnormalities of diabetes cause mitochondrial superoxide overproduction in endothelial cells of both large and small vessels (5). It is considered that not only constant chronic hyperglycemia but also fluctuating blood glucose concentrations $(6,7)$ mediates oxidative stress mechanisms through various pathways under diabetic condition, namely the nonenzymatic glycosylation reaction (8), the electron transport chain in mitochondria and membrane-bound nicotinamide adenine dinucleotide phosphate oxidase (NADPH oxidase) (9). Once formed, free radicals - ROS, reactive nitrogen species (RNS), which are short lived, but highly reactive species trigger cells damage through interaction with a broad range of cell components and biological molecules such as lipids (10), proteins (11) and nucleic acids (12), thus involving in the pathogenesis of distinct pathological conditions.

The aim of this study was to investigate serum levels of various biomarkers of oxidative stress: ROS, nitric oxide radicals $(\bullet \mathrm{NO})$, and those reflecting oxidative damages to proteins, lipids, and deoxyribonucleic acid (protein carbonyl groups, malondialdehyde and 8-hydroxy-2'-deoxyguanosine) in T2DM with vascular complications as compared to healthy subjects, and to determine their predictive value. Further, we have explored their alterations and relationships to disease characteristics.

\section{Materials and Methods}

\subsection{Study subjects}

T2DM patients referred to Endocrinology Clinic of University Hospital "Prof. Dr. St. Kirkovich" in Stara Zagora, Bulgaria, all fulfilled the WHO criteria (13) were included. The study population consisted of 93 type 2 diabetic patients with diabetes-specific complications, 94 healthy controls with normal glucose tolerance tests and absence of a history of either vascular disease or dyslipidemia and 16 newly diagnosed T2DM patients without manifestations of vascular disease.

Clinical and medical history data including obesity based on body mass index $(\mathrm{BMI})>30 \mathrm{~kg} /$ $\mathrm{m}^{2}$; therapy for diabetes, were recorded for each patient. Patients were defined as having suffered a cardiovascular event if they had coronary heart disease, cerebrovascular disease, and/or peripheral artery disease. Diabetic microangiopathy complications (neuropathy, retinopathy and nephropathy) were based on physician diagnosis and/or microalbuminuria, the earliest marker of renal damage, for the latter. A venous blood of patients and controls was collected in the morning after an overnight fasting for lipid profile analysis. Fasting plasma glucose concentrations (FPG) and glycated hemoglobin ( $\mathrm{HbA} 1 \mathrm{c} \%$ ), representing an average measure of glycemic exposure over time, were measured as parameters for glycemic control. Poor glycemic control was taken at HbAlc values > $7 \%$ and FPG $>6.1 \mathrm{mmol} / \mathrm{L}$. Subjects were excluded if they had a history of malignancy, exacerbated cardiac and renal failure, any other hormonal illness, diabetic ketoacidosis, antioxidant supplement intake and/or current active infection. A written informed consent was obtained from each participant and the study was approved by the Ethics Committee.

\subsection{Measurement of oxidative markers}

\subsubsection{Ex vivo electron paramagnetic resonance (EPR) study}

All EPR measurements were performed at room temperature on an X-band EMXmicro, spectrometer Bruker, Germany, equipped with standard Resonator. All EPR experiments were carried out in triplicate and repeated thrice. Spectral processing was performed using Bruker WIN-EPR and Sinfonia software.

\subsubsection{Ex vivo evaluation the levels of ROS products}

The levels of ROS were determined according Shi et al. (14) with some modification. To investigate in real time formation of ROS in the sera of patients and controls was used ex vivo EPR spectroscopy combined with N-tert-butyl-alpha-phenylnitrone (PBN) as a spin trapping agent. $\mathrm{PBN}$, upon reaction with unstable radical such as ROS, forms a relatively stable spin adducts that can be subsequently detected by EPR spectroscopy. Briefly, to $100 \mu \mathrm{L}$ serum was added $900 \mu \mathrm{L} 50 \mathrm{mM}$ PBN dissolved in dimethyl sulfoxide (DMSO) and after centrifugation at 4,000 rpm for 10 min at $4^{\circ} \mathrm{C}$ the EPR spectra were immediately recorded in the supernatant. The levels of ROS products were calculated as double integrated plots of EPR spectra and results were expressed in arbitrary units (arb. units). EPR setting were as follows: $3503.73 \mathrm{G}$ center field, $20.00 \mathrm{~mW}$ microwave power, $5 \mathrm{G}$ modulation amplitude, $50 \mathrm{G}$ sweep width, $1 \times 10^{5}$ gain, $81.92 \mathrm{~ms}$ time constant, $125.95 \mathrm{~s}$ sweep time, 5 scans per sample.

\subsubsection{Ex vivo evaluation the levels of $\bullet N O$ radicals}

Based on the methods published by Yoshioka et al. (15) and Yokoyama et al. (16) we developed and adapted the EPR method for estimation the levels of $\bullet \mathrm{NO}$ radicals in serum. Briefly, to $50 \mu \mathrm{M}$ solution of Carboxy. 
2-(4-Carboxyphenyl)-4,4,5,5-tetramethylimidazoline1-oxyl-3-oxide . potassium salt (PTIO.K) dissolved in a mixture of 50 mMTris $(\mathrm{pH} \mathrm{7.5)}$ and DMSO in a ratio 9:1. To $100 \mu \mathrm{L}$ serum was added $900 \mu \mathrm{L}$ Tris buffer dissolved in DMSO (9:1) after that the mixture is centrifuged at $4000 \mathrm{rpm}$ for $10 \mathrm{~min}$ at $4^{\circ} \mathrm{C} .100 \mu \mathrm{L}$ of sample and $100 \mu \mathrm{L} 50 \mathrm{mM}$ solution of Carboxy. PTIO were mixed and EPR spectra of spin adduct formed between the spin trap Carboxy. PTIO and generated -NO radicals was recorded. The levels of $\bullet$ NO radicals were calculated as double integrated plots of EPR spectra and results were expressed in arbitrary units. The EPR settings were as follows: $3505 \mathrm{G}$ centerfield, $6.42 \mathrm{~mW}$ microwave power, $5 \mathrm{G}$ modulation amplitude, $75 \mathrm{G}$ sweep width, $2.5 \times 10^{2}$ gain, $40.96 \mathrm{~ms}$ time constant, $60.42 \mathrm{~s}$ sweep time, 1 scan per sample.

\subsubsection{Determination of Lipid Peroxidation Products (malondialdehyde)}

Total amount of lipid peroxidation products in the plasma of healthy volunteers and patients was estimated using the thiobarbituric acid (TBA) method of Plaser et al. (17) which measures the malondialdehyde (MDA) reactive products at $532 \mathrm{~nm}$, and results were expressed in $\mu \mathrm{mol} / \mathrm{L}$.

\subsubsection{Protein Carbonyl Content (PCC)}

PCC (nmol/mg) was measured by using a commercial enzyme-linked immunosorbent assay (ELISA) kit followed manufacturer's instructions. The protein carbonyls present in the serum sample or standard are derivatized to 2,4-dinitrophenyl (DNP)hydrazone and probed with an anti-DNP antibody, followed by a horseradish peroxidase (HRP) conjugated secondary antibody. The protein carbonyl content in the serum sample is determined by comparing with a standard curve that is prepared from predetermined reduced and oxidized bovine serum albumin (BSA) standards.

\subsubsection{Quantity of 8-Hydroxy-2'-Deoxyguanosine (8- OHdG)}

The measurement of 8-OHdG was carried out using commercial ELISA kit, followed manufacturer's instructions. The ELISA kit is a competitive enzyme immunoassay developed for rapid detection and quantitation of $8-\mathrm{OHdG}$ in serum. The serum $8-\mathrm{OHdG}$ samples or $8-\mathrm{OHdG}$ standards are coated with BSA conjugated 8-hydroxyguanine (8-OHG) on a preabsorbed microplate. After a brief incubation, an anti-8-OHdG monoclonal antibody is added, followed by a HRP conjugated secondary antibody. The $8-\mathrm{OHdG}$ content in serum samples is determined by comparison with a predetermined $8-\mathrm{OHdG}$ standard curve. The kit has an 8-OHdG detection sensitivity range of $100 \mathrm{pg} / \mathrm{mL}$ to 20 $\mathrm{ng} / \mathrm{mL}$.

\subsection{Statistical analysis}

Data were analyzed using SPSS version 13.0 (SPSS Inc., Chicago, IL, USA). Means with SDs and percentages were calculated to describe clinical and laboratory characteristics of the study subjects. Intergroup comparisons were performed by an analysis of variance (ANOVA), Kruskal-Wallis and Mann-Whitney $U$ tests. Proportional differences were tested using the chi-square $\left(\chi^{2}\right) /$ Fisher's exact tests. Pearson's correlation coefficients were calculated to assess univariate correlations between the continuous parameters. In the cases, where one of the samples is measured as dichotomous, the associations were estimated using the biserial correlation coefficient. Receiver operating characteristic (ROC) analysis was carried out for the evaluation of the diagnostic potential of pro-oxidants for T2DM, associated with vascular complications. The area under the curve (AUC) was calculated, as the diagnostic test that approaches 1 indicates a perfect discrimination. Sensitivity, specificity, positive and negative predictive values according to the cutoff values of the oxidative stress parameters related to diabetes with induced by it vascular changes were calculated. A 2-tailed $p$ values of $<0.05$ were considered statistically significant

\section{Results}

\subsection{Cohort description}

In total, 93 patients with T2DM with diabetes-specific vascular complications (male: female subjects 1: 1.3) aged 30 to 88 years, mean age $64.2 \pm 9.8$ years were studied. The mean disease duration was $12.7 \pm 8.6$ years with a range from 1 to 40 . Approximately half of the diabetics (58.1\%) were obese. Ninety of all patients $(96.8 \%)$ had diabetic neuropathy, 24 (25.8\%) had retinopathy and $7(7.5 \%)$ had nephropathy. The proportion of patients suffering from coronary heart disease was $37.6 \%$. Eleven of the patients (11.8\%) had a medical history of cerebrovascular disease. None of the diabetics was presented with symptomatic lower extremity arterial disease. The diabetics with good glycemic control (39 patients) were taking oral hypoglycemic agents (sulphonylureas and biguanides) $(n=32)$, as in 6 patient biguanides were combined with insulin; and 7 patients were on therapy with insulin alone. In the subgroup of diabetic patients with poor glycemic control $(n=54), 15$ were being treated with oral hypoglycemic agents (sulphonylureas plus biguanides); 39 were insulin-treated; 21 from which with the combination of oral drugs (insulin plus biguanides). Comparison was done with 94 healthy volunteers, 41 men and 53 women, age range from 35 to 83 years, mean age $56.6 \pm 11.2$ years. Only 7 (7.4\%) from the non-diabetic controls had slightly increased bogy weight (BMI 31 - 33). Participants were 
Table 1. General characteristic of the study subjects with T2DM with vascular complications versus healthy controls

\begin{tabular}{|c|c|c|c|}
\hline Variable & Diabetic patients with vascular complications $(n=93)$ & Controls $(n=94)$ & $p$ value \\
\hline Age (years), mean \pm SD & $64.24 \pm 9.76$ & $56.57 \pm 11.19$ & $<0.001$ \\
\hline Sex: male/female & $41 / 52$ & $41 / 53$ & 0.948 \\
\hline Disease duration (years), mean \pm SD & $12.70 \pm 8.65$ & - & - \\
\hline BMI $\left(\mathrm{kg} / \mathrm{m}^{2}\right)$, mean $\pm \mathrm{SD}$ & $31.52 \pm 5.89$ & $25.23 \pm 3.52$ & $<0.001$ \\
\hline BMI $>30 \mathrm{~kg} / \mathrm{m}^{2}, n(\%)$ & $54(58.1)$ & $7(7.4)$ & $<0.001$ \\
\hline $\mathrm{FPG}(\mathrm{mmol} / \mathrm{l})$, mean $\pm \mathrm{SD}$ & $9.52 \pm 5.18$ & $4.97 \pm 0.32$ & $<0.001$ \\
\hline $\mathrm{FPG}>6.1 \mathrm{mmol} / \mathrm{L}, n(\%)$ & $62(66.7)$ & $0(0)$ & - \\
\hline $\mathrm{HbA} 1 \mathrm{c}(\%)$, mean $\pm \mathrm{SD}$ & $8.20 \pm 2.06$ & $5.06 \pm 0.27$ & $<0.001$ \\
\hline $\mathrm{HbAlc}>7 \%, n(\%)$ & $53(57.0)$ & $0(0)$ & - \\
\hline Cholesterol $(\mathrm{mmol} / \mathrm{L})$, mean $\pm \mathrm{SD}$ & $5.12 \pm 1.38$ & $4.43 \pm 0.76$ & $<0.001$ \\
\hline Triglycerides $(\mathrm{mmol} / \mathrm{L})$, mean $\pm \mathrm{SD}$ & $2.43 \pm 1.27$ & $1.52 \pm 0.44$ & $<0.001$ \\
\hline HDL cholesterol $(\mathrm{mmol} / \mathrm{L})$, mean $\pm \mathrm{SD}$ & $1.25 \pm 0.38$ & $1.01 \pm 0.28$ & $<0.001$ \\
\hline LDL cholesterol $(\mathrm{mmol} / \mathrm{L})$, mean $\pm \mathrm{SD}$ & $2.86 \pm 1.10$ & $2.32 \pm 0.62$ & $<0.001$ \\
\hline \multicolumn{4}{|l|}{ Microvascular complications, $n(\%)$} \\
\hline Neuropathy & $90(96.8)$ & - & - \\
\hline Retinopathy & $24(25.8)$ & - & - \\
\hline Nephropathy & $7(7.5)$ & - & - \\
\hline \multicolumn{4}{|l|}{ Macrovascular complications, $n(\%)$} \\
\hline Coronary heart disease & $35(37.6)$ & - & - \\
\hline Cerebrovascular disease & $11(11.8)$ & - & - \\
\hline \multicolumn{4}{|l|}{ Therapy, $n(\%)$} \\
\hline Insulin & $25(26.9)$ & - & - \\
\hline Insulin + oral hypoglycemic agents & $27(29.0)$ & - & - \\
\hline Oral hypoglycemic agents & $41(44.1)$ & - & - \\
\hline ROS [arb. unit], mean \pm SD & $2.01 \pm 0.76$ & $1.19 \pm 1.28$ & $<0.001$ \\
\hline $\operatorname{MDA}[\mu \mathrm{mol} / \mathrm{L}]$, mean $\pm \mathrm{SD}$ & $3.27 \pm 1.01$ & $2.12 \pm 0.55$ & $<0.001$ \\
\hline Protein $\mathrm{CO}$ groups $[\mathrm{nmol} / \mathrm{mg}]$, mean $\pm \mathrm{SD}$ & $8.06 \pm 3.81$ & $3.23 \pm 2.58$ & $<0.001$ \\
\hline 8-OHdG $[\mathrm{ng} / \mathrm{mL}]$, mean $\pm \mathrm{SD}$ & $2.34 \pm 1.19$ & $1.24 \pm 0.51$ & $<0.001$ \\
\hline -NO radicals [arb. unit], mean $\pm \mathrm{SD}$ & $31.22 \pm 3.98$ & $4.30 \pm 5.01$ & $<0.001$ \\
\hline
\end{tabular}

$\mathrm{BMI}=$ body mass index $; \mathrm{FPG}=$ fasting plasma glucose $\mathrm{HbA1c}=$ glycated hemoglobin $; \mathrm{HDL}=$ high density lipids; $\mathrm{LDL}=$ low density lipids; $\mathrm{ROS}=$ reactive oxygen species; arb. unit $=$ arbitrary unit; $\mathrm{MDA}=$ malondialdehyde; 8 -OHdG $=8$-hydroxy-2'-deoxyguanosine; $\bullet \mathrm{NO}$ radicals $=$ nitric oxide radicals.

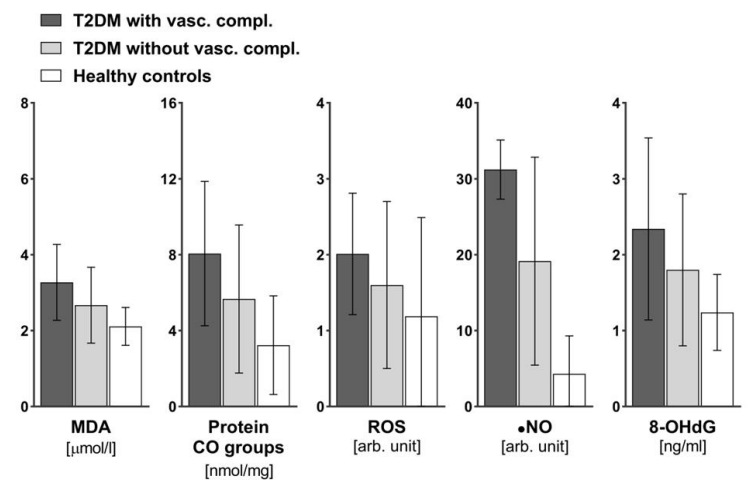

Figure 1. Comparison of the mean serum concentrations of the oxidative markers in T2DM patients with and without vascular complications versus healthy individuals. Shown is increased oxidative capacity in T2DM patients regardless of the presence or absence of vascular disease versus healthy controls. The oxidant profiles among both T2DM subsets demonstrated significantly higher MDA $(p=0.001)$ and $\bullet$ NO radicals $(p$ $<0.001$ ), a tendency of higher protein carbonyl content ( $p=$ 0.09 ) in diabetes with evidence of related vascular involvement and similar levels of ROS and 8-OHdG ( $p=0.147 ; p=0.109$, respectively).

comparable for sex. Also, for comparison purposes, pro-oxidant profiles were examined in a small subset $(n=16)$ of newly diagnosed T2DM patients who had no evidence of complications. Table 1 presents key characteristics of diabetic patients with related vascular disease versus healthy controls.

\subsection{Comparisons of serum levels of pro-oxidants among study subjects}

Oxidative stress levels in circulation, expressed by the 5 biochemical markers, were considerably higher in both T2DM subsets as compared to healthy subjects with numerically higher values in those with signs of vascular damage (Figure 1, Table 1). No difference in serum concentration of all oxidative markers was found among obese and non-obese diabetics with vascular disease $(p>0.05)$. The levels of carbonylated proteins, ROS and 8-OHdG were significantly increased in insufficiently compensated diabetes compared to good glycemic control state $(8.84 \pm 3.95 \mathrm{nmol} / \mathrm{mg} v s .6 .98 \pm$ $3.35 \mathrm{nmol} / \mathrm{mg}, p=0.019 ; 2.14 \pm 0.76$ arb. unit vs. 1.81 \pm 0.73 arb. unit, $p=0.031 ; 3.25 \pm 0.65 \mathrm{ng} / \mathrm{mL} v s .1 .07$ $\pm 0.13 \mathrm{ng} / \mathrm{mL}, p<0.001$, respectively), while MDA and $\bullet \mathrm{NO}$ free radicals were estimated equal in these settings.

3.3. Relationship between serum concentration of oxidative markers and disease characteristics in T2DM patients with associated vascular complications 
Serum MDA showed a weak association with fasting glucose $(r=0.298, p=0.004)$. Calculation of the biserial correlation revealed an inverse correlation of MDA with diagnosed diabetic neuropathy $(r=-0.391, p<0.001)$ and a weak positive correlation of this pro-oxidant with diabetic nephropathy $(r=0.235, p=0.023)$. Protein CO concentrations in the serum correlated weakly with both parameters for glycemic control, fasting plasma glucose and glycated hemoglobin $(r=0.231, p=0.026 ; r=$ $0.260, p=0.012$, respectively). Likewise, systemic levels of the 8 -OHdG showed a good significant correlation with parameters of blood glucose control; with fasting glucose $(r=0.585, p<0.001)$ and with HbA1c $(r=$ $0.697, p<0.001)$. Plasma 8-OHdG levels correlated inversely with the diabetic neuropathy $(r=-0.548, p$ $<0.001)$ and positively with diabetic retinopathy $(r=$ $0.269, p=0.009)$ and history of brain stroke $(r=0.306$, $p=0.003)$. We also found a significant relation between ROS and diabetic retinopathy $(r=0.307, p=0.003)$. No association was established for $\bullet \mathrm{NO}$ radicals with any vascular complication or compensation state of diabetes. Most of the tested pro-oxidants correlated with serum levels of lipids (data not shown).

3.4. Receiver operating characteristic curves with the cutoff values of pro-oxidants for detecting T2DM associated with complications

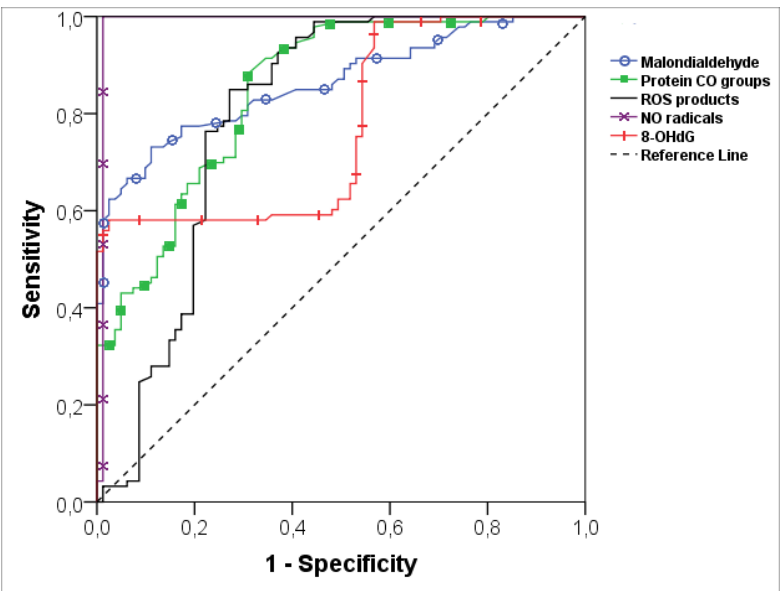

Figure 2. Receiver operating characteristic curves of oxidative biomarkers for detecting T2DM with diabeticinduced vascular damages. The model was performed to distinguish between above-mentioned disease and no disease.
To analyze the predictive accuracy of pro-oxidants for detecting the disease with the best combination of sensitivity and specificity values we calculated ROC. Figure 2 shows the ROC of various pro-oxidants against diabetic condition, with high cutoff values for all biochemical parameters. Significant AUC were obtained for all studied biomarkers $(p<0.001)$. AUC for nitric oxide was very large and substantially bigger compared to those for the other pro-oxidants, approaching a value of 1 , suggesting the greater diagnostic accuracy of this biochemical test (Figure 2, Table 2).

\section{Discussion}

Pro-oxidants generated by chronic exposure to a high glucose concentration or increased glycemic variability and other metabolic abnormalities are important to the development of diabetes with its impairments by modifying biomolecules and cellular damage. Increased free radical generation and subsequent oxidative stress has been implicated as underlying cause of both longterm macrovascular and microvascular complications of diabetes $(4,18)$. In the normal physiological conditions these chemical substances are counterbalanced with the cellular antioxidants, such as superoxide dismutases, catalases and glutathione peroxidases (19). The state of imbalance between pro-oxidants and antioxidants in favor of the former is known as "oxidative stress" (20). Since the vascular damage may begin to develop before the diagnosis and a great proportion of patients with T2DM may already have vascular disease at the time they are diagnosed with diabetes, the main focus of our investigation concerns such a population of patients. In the present study, we have attempted to test which of oxidative stress parameters would improve identification of T2DM with manifested diabetic vascular complications. We analyzed the systemic levels of ROS, $\cdot \mathrm{NO}$ radicals, MDA, protein $\mathrm{CO}$ groups and 8-OHdG basing on the proven role of these reactive molecules and products of biomolecular modification as key tags in the oxidative damage, also on the consideration for the usage of some of them as biomarkers in the diagnosis of diabetes (21).

ROS are chemically reactive molecules, strong oxidants, which by the transfer of their free unpaired

Table 2. Results from ROC analysis

\begin{tabular}{|c|c|c|c|c|c|c|}
\hline Pro-oxidants & Cut-off & AUC & Sensitivity $(\%)$ & Specificity (\%) & PPV (\%) & NPV $(\%)$ \\
\hline $\operatorname{MDA}[\mu \mathrm{mol} / \mathrm{L}]$ & 2.49 & 0.863 & 77.4 & 77.7 & 77.4 & 77.7 \\
\hline Protein $\mathrm{CO}$ groups [nmol/mg] & 5.29 & 0.849 & 71.0 & 72.5 & 72.5 & 71.0 \\
\hline ROS products [arb. unit] & 1.33 & 0.799 & 77.4 & 74.5 & 75.0 & 76.9 \\
\hline •NO radicals [arb. unit] & 17.68 & 0.988 & 100.0 & 98.8 & 98.9 & 100.0 \\
\hline $8-\mathrm{OHdG}[\mathrm{ng} / \mathrm{mL}]$ & 1.44 & 0.773 & 59.1 & 63.0 & 64.7 & 57.3 \\
\hline
\end{tabular}

$\mathrm{AUC}=$ area under the curve; $\mathrm{PPV}=$ positive predictive value; $\mathrm{NPV}=$ negative predictive value; $\mathrm{MDA}=$ malondialdehyde; Protein $\mathrm{CO}$ groups $=$ protein carbonyl groups; ROS $=$ reactive oxygen species; arb. unit $=$ arbitrary unit; $\bullet$ NO radicals $=$ nitric oxide radicals; 8 -OHdG $=8$-hydroxy-2'deoxyguanosine. 
electron cause the oxidation of cellular macromolecules - polyunsaturated fatty acids (PUFAs) in lipids, amino acids in proteins and DNA, resulting in cell structures damage, with subsequent function disruption or cell death (22).

Lipids in cell membrane are among the most susceptible to oxidative damage macro molecules due to the presence of multiple bonds (23). Free radical attack of PUFAs, in particular reaction of oxygen with unsaturated lipids via enzymatic and/or nonenzymatic mechanisms is known as lipid peroxidation. Malondialdehyde (MDA) is one of the final products of lipid peroxidation generated by decomposition of arachidonic acid and larger PUFAs. Evidence now exists to suggest that along with primary dyslipidemia, oxidative damage and irreversible chemical lipoprotein modifications causing a production of oxidized lowdensity lipoproteins (oxLDL) is involved in an accelerated rate of atherosclerosis in T2DM $(24,25)$. Furthermore, this aldehyde (MDA) is also a highly toxic and potentially mutagenic molecule, which exerts these capabilities through its high reactivity with proteins and deoxyribonucleic acids (DNA) $(26,27)$. It is a convenient biomarker for lipid peroxidation and one of the most commonly used in clinical studies (28).

Oxidative modification of proteins is another event of oxidative stress. This oxidative damage often leads to loss in specific protein function and thus might play a significant role in the etiology of diseases. An accumulation and increase in protein carbonyls has been observed in several human pathologies including diabetes mellitus. At present, the measurement of protein $\mathrm{CO}$ groups after their derivatisation with 2,4-dinitrophenylhydrazine (DNPH) is the most widely utilized measure of protein oxidation $(29,30)$. The usage of protein carbonyl $\mathrm{CO}$ groups (aldehydes and ketones) as biomarkers of oxidative stress has some advantages in comparison with the measurement of other oxidation products because of the relative early formation and the relative chemical stability of carbonylated proteins within hours and days (31), whereas lipid peroxidation products undergo detoxification within minutes (32).

A great deal of evidence support the role of oxidative DNA damage affecting tissue function and integrity as a common pathogenic factor in diabetes, as well in the onset and progression of diabetic complications $(33,34)$. 8-hydroxy-2'-deoxyguanosine (8-OHdG), an oxidized nucleoside of nuclear and mitochondrial DNA, is a sensitive DNA damage biomarker of cellular oxidative stress. Diverse pathologies affect $8-\mathrm{OHdG}$ level, but it is considered to be a measurable risk factor for cancer, atherosclerosis, and diabetes (34), such that its use as a biomarker in the diagnosis of diabetes has been considered (21).

-NO is a common gaseous free radical, which plays a role in vascular physiology and is also, known as endothelium derived relaxing factor. Therefore reduction of NO production, release or decreased bioavailability to the endothelium leads to endothelial cell dysfunction (35). Endothelial dysfunction plays an important role in the early stages of development of atherosclerosis, which is often observed as a macroangiopathic event in T2DM (36).

The results from our analysis showed a considerable overproduction of the 5 studied oxidative markers in T2DM patients, more distinctly in the presence of diabetes-associated vascular manifestations. These results are in agreement with the majority of publications, which demonstrate a significant rise in oxidative damage in the diabetic state $(3,4,37-39)$. Notably, our T2DM patients with vascular involvement significantly exhibit approximately 7-fold elevated -NO levels than healthy controls, unlike the other biochemical markers which were increased only by 1.5 to 2.5 times, suggesting, that $\cdot \mathrm{NO}$ free radical overproduction may be considered crucial in the generation of diabetes and vascular disease. The glycemic control status was significantly correlated to the level of oxidation of the lipids, proteins and DNAs (MDA, carbonyl values and 8-OHdG), which confirms the influence of elevated blood glucose on the damage of all types of biological molecules. The strongest degrees of correlation were apparent for the oxidized guanine of DNA, showing that hyperglycemia promotes DNA oxidation. ROS and $8-\mathrm{OHdG}$ generated by high glucose were causally linked to diabetic retinopathy. Correspondingly, a positive correlation of the systemic 8-OHdG content with the brain stoke, a macrovascular manifestation attributable to diabetes was found. However, deoxyribonucleic acid base modification produced by the oxidation of deoxyguanosine was inversely correlated with diabetic neuropathy. MDA was associated with various locations of microvascular damage (neuro- and nephropathy) with a divergent link orientation. We further demonstrated the lack of difference in the systemic oxidative stress among obese and non-obese diabetics, despite the opposite findings of the other authors for the increase oxidative stress on accumulated fat in obesity (40).

ROC analysis revealed that $\cdot \mathrm{NO}$ values in the circulation discriminated the presence of T2DM with diabetes-specific vascular complications better than the other oxidative stress parameters. The cutoff values based on the ROC were higher and outside the normal range for all of them. $8-\mathrm{OHdG}$ had lower sensitivity (0.59) and specificity (0.63) compared with the other biomarkers and showed a reduced AUC, while the -NO gives the highest returns for detecting diabetes with induces vascular damage. In general, the test characteristics positive and negative predictive values were in favor of the $\mathrm{NO}$ radicals $(98.9 \%$ and $100 \%$, respectively) while the positive predictive value of 8-OHdG variable was low (only 64.7 percent). Overall the positive predictive values - a relevant characteristic 
in clinical practice - for MDA, carbonylated proteins and ROS were uniformly reasonable.

In conclusion, the chronic excess of carbohydrates present in T2DM and poor metabolic control result in creation of an abundance of reactive intermediates, leading to increase chemical modification of proteins, lipids and DNAs. Oxidative stress affects vascular function and ultimately contributes to vascular disease. The cutoff value for the studied oxidative biomarkers obtained from plotted ROC curves may serve as an indicator of clinical reference for detecting T2DM with associated vascular complications. The most efficient indicator is $\bullet \mathrm{NO}$, which may have the added ability to account for the disease and may strengthen the accuracy of clinical diagnosis.

\section{Acknowledgement}

This study was supported by scientific projects 6/2016 of Medical Faculty, Trakia University, Stara Zagora, Bulgaria.

\section{References}

1. DeFronzo RA. The Triumvirate: $\beta$-Cell, Muscle, Liver: A Collusion Responsible for NIDDM. Diabetes. 1988; 37:667-687.

2. Wallace JI. Management of Diabetes in the Elderly. Clin Diabetes. 1999; 17:1-26.

3. Evans JL, Goldfine ID, Maddux BA, Grodsky GM. Oxidative stress and stress-activated signaling pathways: A unifying hypothesis of type 2 diabetes. Endocr Rev. 2002; 23:599-622.

4. Rösen P, Nawroth PP, King G, Möller W, Tritschler HJ, Packer L. The role of oxidative stress in the onset and progression of diabetes and its complications: A summary of a Congress Series sponsored by UNESCO-MCBN, the American Diabetes Association and the German Diabetes Society. Diabetes Metab Res Rev. 2001; 17:189-212.

5. Giacco F, Brownlee M. Oxidative stress and diabetic complications. Circ Res. 2010; 107:1058-1070.

6. Hirsch IB, Brownlee M. Should minimal blood glucose variability become the gold standard of glycemic control? J Diabetes Complications. 2005; 19:178-181.

7. Wright E Jr, Scism-Bacon JL, Glass LC. Oxidative stress in type 2 diabetes: The role of fasting and postprandial glycaemia. Int J Clin Pract. 2006; 60:308-314.

8. Sakurai T, Tsuchiya S. Superoxide production from nonenzymatically glycated protein. FEBS Lett. 1988; 236:406-410.

9. Harrison D, Griendling KK, Landmesser U, Hornig B, Drexler H. Role of oxidative stress in atherosclerosis. Am J Cardiol. 2003; 91:7-11.

10. Biliński T, Litwińska J, Błaszczyński M, Bajus A. Superoxide dismutase deficiency and the toxicity of the products of autooxidation of polyunsaturated fatty acids in yeast. Biochim et Biophys Acta. 1989; 1001:102-106.

11. Cabiscol E, Piulats E, Echave P, Herrero E, Ros J. Oxidative stress promotes specific protein damage in Saccharomyces cerevisiae. J Biol Chem. 2000; 275:2739327398.
12. Yakes FM, Van Houten B. Mitochondrial DNA damage is more extensive and persists longer than nuclear DNA damage in human cells following oxidative stress. Proc Natl Acad Sci USA. 1997; 94:514-519.

13. World Health Organization \& International Diabetes Federation. Definition and diagnosis of diabetes mellitus and intermediate hyperglycaemia: Report of a WHO/IDF consultation. Geneva, 2006: World Health Organization. http://www.who.int/iris/handle/10665/43588. (accessed January 5, 2019).

14. Shi H, Sui Y, Wang X, Luo Y, Ji L. Hydroxyl radical production and oxidative damage induced by cadmium and naphthalene in liver of Carassius auratus Comp. Biochem and Physiol. Part C: Toxicol and Pharmacol. 2005; 140:115-121.

15. Yoshioka T, Iwamoto N, Lto K. An applicationof Electron Paramagnetic Resonance to evaluate nitric oxide and its quenchers. J Am Soci Nephrol. 1996; 7:961-965.

16. Yokoyama K, Hashiba K, Wakabayashi H, Hashimoto K, Satoh K, Kurihara T, Motohashi N, Sakagami H. Inhibition of LPS-stimulated NO production in mouse macrophage-like cells by Tropolones. Anticans Res. 2004; 24:3917-3922.

17. Plaser ZA, Cushman LL, Jonson BC. Estimation of product of lipid peroxidation (malonyl dialdehyde) in biochemical systems. Analyt Biochem. 1966; 16:359-364.

18. Pham-Huy LA, He H., Pham-Huy C. Free radicals, antioxidants in disease and health. IJBS. 2008; 4:89-96.

19. Gutteridge JM, Halliwell B. Comments on review of Free Radicals in Biology and Medicine, by Barry Halliwell and John MC. Gutteridge. Free Radic Biol Med. 1992; 12:9395.

20. Sies H. Oxidative stress: Oxidants and antioxidants. Exp Physiol. 1997; 82:291-295.

21. Lai CQ, Tucker KL, Parnell LD, Adiconis X, GarcíaBailo B, Griffith J, Meydani M, Ordovás JM. PPARGC1A variation associated with DNA damage, diabetes, and cardiovascular diseases. Diabetes. 2008; 57:809-816.

22. Halliwell B. Antioxidants in human health and disease. Ann Rev Nutr. 1996; 16:33-50.

23. Butterfield DA, Koppal T, Howard B, Subramaniam RA, Hall N, Hensley K, Yatin S, Allen K, Aksenov M, Aksenova M, Carney J. Structural and functional changes in proteins induced by free radical-mediated oxidative stress and protective action of the antioxidants N-tertbutyl-alpha-phenylnitrone and vitamin E. Ann N Y Acad Sci. 1998; 854:448-462.

24. Steinberg D, Parthasarathy S, Carew TE. Beyond cholesterol. Modifications of low-density lipoprotein that increases its atherogenicity. New Engl J Med. 1989; 320:915-924.

25. Jenkins AJ, Best JD, Klein RL, Lyons TJ. Lipoproteins, glycoxidation and diabetic angiopathy. Diabetes Metab Res Rev. 2004; 20:349-368.

26. Esterbauer H, Eckl P, and Ortner A. Possible mutagens derived from lipids and lipid precursors. Mutation Research. 1990; 238:223-233.

27. Del Rio D, Stewart AJ, Pellegrini N. A review of recent studies on malondialdehyde as toxic molecule and biological marker of oxidative stress. Nutr Metab Cardiovasc Dis. 2005; 15:316-328.

28. Giera M, Lingeman H, Niessen WMA. Recent Advancements in the LC- and GC-Based Analysis of Malondialdehyde (MDA): A Brief Overview. Chromatographia. 2012; 75:433-440. 
29. Chevion M, Berenshtein E, Stadtman ER. Human studies related to protein oxidation: Protein carbonyl content as a marker of damage. Free Radic Res. 2000; 33:99-108.

30. Beal MF. Oxidatively modified proteins in aging and disease. Free Radic Biol Med. 2002; 32:797-803.

31. Grune T, Reinheckel T, Davies KJA. Degradation of oxidized proteins in K562 human hematopoietic cells by proteasome. J Biol Chem. 1996; 271:15504-15509.

32. Siems WG, Zollner H, Grune T, Esterbauer H. Metabolic fate of 4-hydroxynonenal in hepatocytes: 1,4-dihydroxynonene is not the main product. J Lipid Res. 1997; 38:612-622.

33. Ayepola OR, Brooks NL, Oguntibeju OO. Oxidative Stress and Diabetic Complications: The Role of Antioxidant Vitamins and Flavonoids. In: AntioxidantAntidiabetic Agents and Human Health. InTech, Croatia, 2014; pp. 25-58

34. Wu LL, Chiou CC, Chang PY, Wu JT. Urinary 8-OHdG: A marker of oxidative stress to DNA and a risk factor for cancer, atherosclerosis and diabetics. Clin Chim Acta. 2004; 339:1-9.

35. Liao JK. Endothelium and acute coronary syndromes. Clin Chem. 1998; 44:1799-1808.
36. Cai H, Harrison DG. Endothelial dysfunction in cardiovascular diseases: The role of oxidant stress. Circ Res. 2000; 87:840-844.

37. Nakhjavani M, Esteghamati A, Nowroozi S, Asgarani F, Rashidi A, Khalilzadeh O. Type 2 diabetes mellitus duration: An independent predictor of serum malondialdehyde levels. Singapore Med J. 2010; 51:582585.

38. Bollineni RC, Fedorova M, Blüher M, Hoffmann R. Carbonylated plasma proteins as potential biomarkers of obesity induced type 2 diabetes mellitus. J Proteome Res. 2014; 13:5081-5093.

39. Pan HZ, Zhang H, Chang D, Li H, Sui H. The change of oxidative stress products in diabetes mellitus and diabetic retinopathy. Br J Ophthalmol. 2008; 92:548-551.

40. Furukawa S, Fujita T, Shimabukuro M, Iwaki M, Yamada Y, Nakajima Y, Nakayama O, Makishima M, Matsuda $\mathrm{M}$, Shimomura I. Increased oxidative stress in obesity and its impact on metabolic syndrome. J Clin Invest. 2004; 114:1752-1761.

(Received January 25, 2019; Revised March 11, 2019; Accepted March 21, 2019) 